\title{
BANACH SPACE BIFURCATION THEORY
}

BY

\section{DAVID WESTREICH}

ABSTRACT. We consider the bifurcation problem for the nonlinear operator equation $x=\lambda L x+T(\lambda, x, y)$ in a real Banach space $X$. Here $\lambda_{0}$ is an eigenvalue of the bounded linear operator $L, X=N\left(I-\lambda_{0} L\right) \oplus R\left(I-\lambda_{0} L\right), T \in C^{i}$ and $T$ is of higher order in $x$.

New techniques are developed to simplify the solution of the bifurcation problem. When $\lambda_{0}$ is a simple eigenvalue, $\lambda_{0}$ is shown to be a bifurcation point of the homogeneous equation (i.e. $y \equiv 0$ ) with respect to 0 . All solutions near $\left(\lambda_{0}, 0\right)$ are shown to be of the form $(\lambda(\epsilon), x(\epsilon)), 0 \leq|\epsilon|<\epsilon_{0}, \lambda(\epsilon)$ and $x(\epsilon)$ are continuous and $\lambda(\epsilon)$ and $x(\epsilon)$ are in $C^{n}$ or real analytic as $T$ is in $C^{n+1}$ or is real analytic. When $T$ is real analytic and $\lambda(\epsilon) \not \lambda_{0}$ then there are at most two solution branches, and each branch is an analytic function of $\lambda$ for $\lambda \neq \lambda_{0}$. If $T$ is odd and analytic, for each $\lambda \in\left(\lambda_{0}-\delta, \lambda_{0}\right)$ (or $\lambda \in\left(\lambda_{0}, \lambda_{0}+\delta\right)$ ) there exist two nontrivial solutions near 0 and there are no solutions near 0 for $\lambda \in\left(\lambda_{0}, \lambda_{0}+\delta\right)\left(\right.$ or $\left.\lambda \in\left(\lambda_{0}-\delta, \lambda_{0}\right)\right)$.

We then demonstrate that in each sufficiently small neighborhood of a solution of the homogeneous bifurcation problem there are solutions of the nonhomogeneous equation (i.e. $y \not 0$ ) depènding continuously on a real parameter and on $y$.

If $\lambda_{0}$ is an eigenvalue of odd multiplicity we prove it is a point of bifurcation of the homogeneous equation.

With a strong restriction on the projection of $T$ onto the null space of $I-\lambda_{0} L$ we show $\lambda_{0}$ is a bifurcation point of the homogeneous equation when $\lambda_{0}$ is a double eigenvalue.

Counterexamples to some of our results are given when the hypotheses are weakened.

Introduction. In this paper we investigate the bifurcation problem for a nonlinear operator equation in a real Banach space. We seek solutions $(\lambda, x, y)$ near $\left(\lambda_{0}, 0,0\right)$ of the equation

$$
(I-\lambda L) x-T(\lambda, x, y)=0
$$

where $L$ is a bounded linear operator and $T$ is continuously differentiable and of higher order in $x$, and $\lambda_{0}$ is an eigenvalue of $L$. When the null space of $I-\lambda_{0} L$ is finite-dimensional we assume $I-\lambda_{0} L$ is a Fredholm operator of index 0 . $I-\lambda_{0} L$ satisfies a comparable hypothesis when the null space is infinite-dimensional.

Krasnosel'skiu [5] considered the homogeneous equation (where $y \equiv 0$ ) for $\lambda_{0}$

Received by the editors May 3, 1971 and; in revised form, December 8, 1971.

AMS 1970 subject classifications. Primary 47H15; Secondary 46N05.

Key words and phrases. Banach space, bifurcation, homogeneous equation, nonhomo* geneous equation, Fredholm operator, index zero, nonlinear operator, Fréchet differentiable, real analytic, symmetric $n$-linear form, simple eigenvalue, eigenvalue of odd multiplicity, implicit function theorem. 
a simple eigenvalue. He assumed that $T$ depends linearly on $\lambda$, and made further assumptions regarding the projection of $T(x)$ onto the null space of $I-\lambda_{0} L$. With these restrictions he was able to prove that $\lambda_{0}$ is a bifurcation point of the equation with respect to 0 .

We can dispense with these additional hypotheses and yet obtain the same conclusion for the homogeneous case when $\lambda_{0}$ is a simple eigenvalue.

Using simple methods based on the implicit function theorem, we develop the complete solution of the bifurcation problem when $\lambda_{0}$ is a simple eigenvalue. These same techniques also enable us to prove that if $\lambda_{0}$ is an eigenvalue of odd multiplicity then $\lambda_{0}$ is a bifurcation point of the homogeneous equation.

Ir Part I we introduce several simplifications of the bifurcation problem. We show that the problem is equivalent to finding solutions of a related operator equation in the null space of $I-\lambda_{0} L$. For the homogeneous case we prove the existence of a unique function, $\hat{\lambda}(u)$, expressing $\lambda$ as a function of the vectors in the null space. The function $\hat{\lambda}(u)$ has the property that if $(\lambda, u, 0)$ is a solution of the related equation thep $\lambda=\hat{\lambda}(u)$.

The solution of the bifurcation problem when $\lambda_{0}$ is a simple eigenvalue is developed in Part II. The solutions $(\lambda, x, 0)$ of the homogeneous equation are shown to be a continuous function of a real parameter, varying in a neighborhood of 0 . This function is $n$ times continuously differentiable or real analytic as $T$ is $n+1$ times continuously differentiable or real analytic. When $T$ is real analytic and $\lambda_{0}$ is not the only eigenvalue of the nonlinear equation, there exists at most two distinct solution branches depending continuously on $\lambda$, and each branch is analytic in a deleted neighborhood of $\lambda_{0}$.

We proceed from the homogeneous case to the nonhomogeneous case. We demonstrate that in each sufficiently small neighborhood of a solution of the homogeneous equation there are solutions of the nonhomogeneous equation depending continuously on a real parameter and on $y$.

Our methods do not yield any results for the nonhomogeneous equation when $\lambda \equiv \lambda_{0}$. For this important case the reader is referred to the survey article of Vainberg and Trenogin [7] and the references cited therein.

When $\lambda_{0}$ is an eigenvalue of higher multiplicity the bifurcation problem for the homogeneous equation becomes more difficult, and in general $\lambda_{0}$ is not a point of bifurcation. Nevertheless, some results for $\lambda_{0}$ of higher multiplicity have been obtained. Krasnosel'skil [4] showed that if $L+T$ is compact and is a gradient operator depending linearly on $\lambda$ then $\lambda_{0}$ is a point of bifurcation. He also showed, by means of the degree of a mapping, that if $L+T$ is compact and $\lambda_{0}$ is an eigenvalue of odd multiplicity then $\lambda_{0}$ is a bifurcation point.

In Part III we show that under our hypotheses, if $\lambda_{0}$ is an eigenvalue of odd multiplicity then $\lambda_{0}$ is a point of bifurcation of the homogeneous equation with 
respect to 0 . We thus eliminate the restrictions that $L+T$ be compact and $T$ depend linearly on $\lambda$.

In addition, by imposing the strong restriction on $T$ that one of the components of the projection of $T$ onto the null space of $I-\lambda_{0} L$ is of constant sign, we show that if $\lambda_{0}$ is a double eigenvalue then $\lambda_{0}$ is a bifurcation point of the homogeneous equation with respect to 0 .

Part IV includes counterexamples to some of our results when the hypotheses are weakened. One of the examples shows that if $\lambda_{0}$ is not simple and the dimension of the null space of $I-\lambda_{0} L$ is one, then $\lambda_{0}$ may fail to be a bifurcation point.

This paper is based on a part of the author's dissertation submitted to the Belfer Graduate School of Sciences, Yeshiva University in candidacy for the degree of Doctor of Philosophy. The author expresses his thanks to Professor M. S. Berger for constant encouragement and numerous helpful suggestions.

\section{PART I. THE BIFURCATION PROBLEM AND ITS REDUCTION}

1.1. In this part we introduce the general bifurcation problem and develop techniques which facilitate its solution.

Let $X$ and $Y$ be real Banach spaces, $L$ a bounded linear map of $X$ into $X$ and let $\lambda_{0}$ be an eigenvalue of $L$. Suppose we perturb $L$ by adding a nonlinear operator $T$ which is continuous in a neighborhood of $\left(\lambda_{0}, 0,0\right)$ in $\mathbf{R} \times X \times Y$ with range in $X$. We seek solutions $(\lambda, x, y)$ of the equation

$$
(I-\lambda L) x-T(\lambda, x, y)=0 .
$$

for $(\lambda, x, y)$ near $\left(\lambda_{0}, 0,0\right)$.

We make the following assumptions about $L$ and $T$ :

H1. $R\left(I-\lambda_{0} L\right)$ is closed and $X=N\left(I-\lambda_{0} L\right) \oplus R\left(I-\lambda_{0} L\right)$, where $R\left(I-\lambda_{0} L\right)$ denotes the range of $I-\lambda_{0} L$ and $N\left(I-\lambda_{0} L\right)$ its kernel.

H2. $T(\lambda, 0,0)=0$ for all $\lambda$ near $\lambda_{0}$. T is continuously Fréchet differentiable in a neighborhood of $\left(\lambda_{0}, 0,0\right)$ and $D_{2} T(\lambda, 0,0) \equiv 0$ for all $\lambda$ near $\lambda_{0}$.

When $N\left(I-\lambda_{0} L\right)$ is a finite-dimensional subspace of $X$ then $\mathrm{H} 1$ is equivalent to the assumption $N\left(I-\lambda_{0} L\right) \cap R\left(I-\lambda_{0} L\right)=\{0\}$ and $I-\lambda_{0} L$ is a Fredholm operator of index 0 (see appendix for the proof).

There are many instances when condition $\mathrm{H} 1$ is met. $\mathrm{Hl}$ is satisfied when, for example, $L$ is compact and $N\left(I-\lambda_{0} L\right) \cap R\left(I-\lambda_{0} L\right)=\{0\}$; or if $X$ is a Hilbert space and $L$ is a compact selfadjoint map.

In a later section it will be convenient to assume $T$ is real analytic. For symmetric $n$-linear forms [6, p. 179], [7], we set

$$
a_{n}\left(x_{1}, \cdots, x_{1}, \frac{x_{2}, \cdots, x_{2}}{n_{1} \text { times }}, \cdots, \frac{x_{k}, \cdots, x_{k}}{n_{2} \text { times }}\right)=a_{n}\left(x_{1}^{n_{1}}, x_{2}^{n_{2}}, \cdots, x_{k}^{n_{k}}\right) .
$$


We then say an operator $T$ is real analytic at a point $x_{0}$ if it is infinitely differentiable at $x_{0}$ and, in some neighborhood of $x_{0}$, is expressible as a Taylor series

$$
T(x)=\sum_{n=0}^{\infty} a_{n}\left(x_{0} ;\left(x-x_{0}\right)^{n}\right)
$$

where each $a_{n}$ is a continuous symmetric $n$-linear form. An operat or $T$ is called real analytic if it is real analytic at each point of its domain.

We shall make essential use of the following form of the implicit function theorem.

Theorem 1.1 (implicit function theorem). Let $X, Y$, and $Z$ be Banach spaces, $\left(x_{0}, y_{0}\right) \in X \times Y$ and let $T$ be a continuous map of a neigbborbood $A$ of $\left(x_{0}, y_{0}\right)$ into $Z$ such that $D_{2} T$ exists and is continuous in $A$. Suppose $T\left(x_{0}, y_{0}\right)$ $=0$ and $D_{2} T\left(x_{0}, y_{0}\right)$ is a linear homeomorphism of $Y$ onto $Z$. Then there is an open neighborbood $U$ of $x_{0}$ and an open neighborbood $V$ of $y_{0}$ such that the following are true:

(i) There exists a unique continuous function $f$ mapping $U$ into $V$ such that $T(x, f(x))=0$ for $x \in U$.

(ii) If for $x \in U$ and $y \in V, T(x, y)=0$ then $f(x)=y$.

(iii) If $T \in C^{1}(A)$ then $f \in C^{1}(U)$ and the derivative of $f$ is given by

$$
D f(x)=-\left(D_{2} T(x, f(x))\right)^{-1} \circ D_{1} T(x, f(x)) .
$$

(iv) If $T \in C^{n}(A)$ then $f \in C^{n}(U)$.

(v) If $T$ is real analytic in $A$ then $f$ is real analytic in $U$.

Proof. The proof is a straightforward application of the contraction mapping principle. (See [3, pp. 194-196], [6, pp. 265-269] and [7].)

The problem of finding solutions of equation (1.1) can be reduced to finding solutions of an equation in $N\left(I-\lambda_{0} L\right)$. If $\operatorname{dim} N\left(I-\lambda_{0} L\right)<\infty$ the original problem will thus be simplified to a finite-dimensional one. We now proceed with this reduction.

By H1, if $x \in X, x=u+v$ where $u \in N\left(I-\lambda_{0} L\right)$ and $v \in R\left(I-\lambda_{0} L\right)$ and there exist canonical projections $P$ and $P^{*}$ of $X$ onto $N\left(I-\lambda_{0} L\right)$ and $X$ onto $R\left(I-\lambda_{0} L\right)$ respectively, such that $P(x)=P(u+v)=u$ and $P^{*}(x)=P^{*}(u+v)=v$.

Since the operators $I-\lambda L$ and $I-\lambda_{0} L$ commute, $R\left(I-\lambda_{0} L\right)$ and $N\left(I-\lambda_{0} L\right)$ are invariant under $I-\lambda L$. Thus we see that for $u \in N\left(I-\lambda_{0} L\right)$ and $v \in R\left(I-\lambda_{0} L\right), P(I-\lambda L)(u+v)=(I-\lambda L) u$ and $P^{*}(I-\lambda L)(u+v)=(I-\lambda L) v$. Therefore, $x=u+v$ is a solution of equation (1.1) for $\lambda$ and $y$ if and only if for $\lambda$ and $y, u$ and $v$ are a solution of the system of equations

$$
\begin{gathered}
(I-\lambda L) u-P T(\lambda, u+v, y)=0, \\
(I-\lambda L) v-P^{*} T(\lambda, u+v, y)=0 .
\end{gathered}
$$


Using the implicit function theorem we show that for each $(\lambda, u, y)$ near $\left(\lambda_{0}, 0,0\right)$ equation (1.3) always has a unique solution $v$ in $R\left(I-\lambda_{0} L\right)$. For convience we denote $N\left(I-\lambda_{0} L\right)$ by $\pi$.

Theorem 1.2. There exist a neigbborbood $A$ of $\left(\lambda_{0}, 0,0\right)$ in the product space $\mathbf{R} \times \Re \times Y$, a neigbborbood $B$ of 0 in $R\left(I-\lambda_{0} L\right)$, and a unique continuous map $f$ of $A$ into $B$, such that the following are true:

(i) $(I-\lambda L) f(\lambda, u, y)-P^{*} T(\lambda, u+f(\lambda, u, y), y)=0$ for all $(\lambda, u, y) \in A$.

(ii) If $(\lambda, u, y) \in A$ and $v \in B$ satisfy equation (1.3), then $f(\lambda, u, y)=v$.

(iii) $f \in C^{1}$ and

$$
D f(\lambda, u, y)=-\left(D_{2} F(z, f(z))\right)^{-1} \circ D_{1} F(z, f(z))
$$

where $z=(\lambda, u, y)$ and

$$
F((\lambda, u, y), v)=(I-\lambda L) v-P^{*} T(\lambda, u+v, y) .
$$

(iv) If $T \in C^{n}$ then $f \in C^{n}$.

(v) If $T$ is real analytic then $f$ is real analytic.

Proof. By H1, $I-\lambda_{0} L$ is a 1-1 map of $R\left(I-\lambda_{0} L\right)$ onto $R\left(I-\lambda_{0} L\right)$. Then by the bounded inverse theorem, $I-\lambda_{0} L$ is also a linear homeomorphism. The operator $F$ defined by equation (1.5) is a continuous map of an open neighborhood of $\left(\left(\lambda_{0}, 0,0\right), 0\right) \in(\mathbf{R} \times \Re \times Y) \times R\left(I-\lambda_{0} L\right)$ into $R\left(I-\lambda_{0} L\right) . \quad F\left(\left(\lambda_{0}, 0,0\right), 0\right)=0$, $D_{2} F$ is continuous and $D_{2} F\left(\left(\lambda_{0}, 0,0\right), 0\right)=I-\lambda_{0} L$. The results then follow immediately from the implicit function theorem.

Corollary 1.3. If $(\lambda, u, y)$ near $\left(\lambda_{0}, 0,0\right)$ in $\mathbf{R} \times N\left(I-\lambda_{0} L\right) \times Y$ satisfies the equation

$$
(I-\lambda L) u-P T(\lambda, u+f(\lambda, y), y)=0
$$

then $(\lambda, u+f(\lambda, u, y), y)$ is a solution of equation (1.1). Conversely, if $(\lambda, x, y)$ near $\left(\lambda_{0}, 0,0\right)$ satisfies equation (1.1), then, if $x=u+v,(\lambda, u, y)$ is a solution of equation (1.6) and $v=f(\lambda, u, y)$.

Proof. The proof of this corollary follows by Theorem 1.2 and our previous discussion.

Corollary 1.3 implies that the solutions of small norm of equation (1.1) for $\lambda$ and $y$ near $\lambda_{0}$ and 0 are completely determined by the solutions of small norm of equation (1.6).

Definition. Let $\left(\lambda_{0}, 0\right)$ be a point in $\mathbf{R} \times X$ and $K$ a continuous map of a neighborhood of $\left(\lambda_{0}, 0\right)$ into $Y$. Suppose $K(\lambda, 0)=0$ for all $\lambda$ near $\lambda_{0}$. The real number $\lambda_{0}$ is called a bifurcation point of the equation $K(\lambda, x)=0$ with respect to 0 if, for any $\epsilon, \delta>0$ there exist a real number $\lambda$ and a nonzero vector $x \in X$ such that $K(\lambda, x)=0$ and $0 \leq\left|\lambda-\lambda_{0}\right|<\epsilon$ and $\|x\|<\delta$. 
Corollary 1.4. $\lambda_{0}$ is a bifurcation point of the bomogeneous equation

$$
(I-\lambda L) x-T(\lambda, x, 0)=0
$$

with respect to 0 if and only if $\lambda_{0}$ is a bifurcation point of the bomogeneous equation

$$
(I-\lambda L) u-P T(\lambda, u+f(\lambda, u, 0), 0)=0
$$

with respect to 0 .

Proof. This fact follows from Corollary 1.3.

1.2. We impose two additional assumptions on $T$. With these hypotheses a further simplification of our problem is possible. Let $T(\lambda, x)=T(\lambda, x, 0)$ and $f(\lambda, u)=f(\lambda, u, 0)$.

H3. Either $D_{2} T(\lambda, x) \rightarrow 0$ uniformly for $\lambda$ near $\lambda_{0}$ as $x \rightarrow 0$, or $\operatorname{dim} N\left(I-\lambda_{0} L\right)<\infty$.

H4. $\left\|D_{1} T(\lambda, x)\right\|=o(\|x\|)$ uniformly for $\lambda$ near $\lambda_{0}$.

Remark. If $T$ is either of the form $T(\lambda, x)=\lambda T(x)$ or $T(\lambda, x)=T(x)$, it is unnecessary to assume $\mathrm{H} 3$ and $\mathrm{H} 4$, since they are consequencès of $\mathrm{H} 2$ and the definition of the derivative.

The following lemma will be used in the proof of Lemma 1.6. Its proof will be given in the appendix.

Lemma 1.5. Let $\left(x_{0}, 0\right) \in X \times Y$ and $T$ be a continuous map of a neighborbood of $\left(x_{0}, 0\right)$ into $Z$.

(i) If $T(x, 0)=0$ for all $x$ and $D_{2} T$ is continuous, then as $y \rightarrow 0, T(x, y)$ $\rightarrow 0$ uniformly for all $x$ in a neighborbood of $x_{0}$.

(ii) If, in addition, as $y \rightarrow 0, D_{2} T(x, y) \rightarrow 0$ uniformly for $x$ in a neighborbood of $x_{0}$, then $\|T(x, y)\|=o(\|y\|)$ uniformly for $x$ in a neighborbood of $x_{0}$.

With $\mathrm{H} 3$ and $\mathrm{H} 4$ we prove the following lemma:

Lemma 1.6. There exists an interval $A$ about $\lambda_{0}$ such that the following are true:

(i) $\|f(\lambda, u)\|=o(\|u\|)$ uniformly for $\lambda \in A$.

(ii) $\|T(\lambda, u+f(\lambda, u))\|=o(\|u\|)$ uniformly for $\lambda \in A$.

(iii) $\left\|D_{1} f(\lambda, u)\right\|=o(\|u\|)$ uniformly for $\lambda \in A$.

Proof. By Theorem 1.2, $f(\lambda, 0)=0$ for $\lambda$ near $\lambda_{0}$ and $D_{2} f$ is continuous. Lemma 1.5 implies $f(\lambda, u) \rightarrow 0$ uniformly for $\lambda$ near $\lambda_{0}$ as $u \rightarrow 0$. It is well known that a continuous map of a closed bounded subset of a finite-dimensional Banach space into a Banach space is uniformly continuous. Hence, by $\mathrm{H} 3$, $D_{2} T(\lambda, u+f(\lambda, u)) \rightarrow 0$ uniformly for $\lambda$ near $\lambda_{0}$ as $u \rightarrow 0$.

By equations (1.4) and (1.5), 


$$
D_{2} f(\lambda, u)=-\left[D_{2} F((\lambda, u), f(\lambda, u))\right]^{-1} \circ\left[-D_{2} P^{*} T(\lambda, u+f(\lambda, u))\right]
$$

where $F((\lambda, u), v)=(I-\lambda L) v-P^{*} T(\lambda, u+v)$. From equation (1.9) we get the inequality

$$
\left\|D_{2} f(\lambda, u)\right\| \leq\left\|\left[D_{2} F((\lambda, u), f(\lambda, u))\right]^{-1}\right\|\left\|D_{2} P^{*} T(\lambda, u+f(\lambda, u))\right\| \cdot
$$

By cont inuity there is a neighborhood of $\left(\lambda_{0}, 0\right)$ for which $\left\|\left[D_{2} F((\lambda, u), f(\lambda, u))\right]^{-1}\right\|$ is uniformly bounded. Thus there is a neighborhood $A$ about $\lambda_{0}$ for which $D_{2} f(\lambda, u) \rightarrow 0$ uniformly for $\lambda \in A$ as $u \rightarrow 0$. Statement (i) then follows by Lem ma 1.5.

To show statement (ii) we note that the partial derivative of $T(\lambda, u+f(\lambda, u))$ with respect to $u$ goes to 0 uniformly for $\lambda \in A$ as $u \rightarrow 0$. The result then follows from Lemma 1.5.

We now prove statement (iii). By equation (1.4) $D_{1} f(\lambda, u)$

$$
=-\left[D_{2} F((\lambda, u), f(\lambda, u))\right]^{-1} \circ\left[D_{1}((I-\lambda L) f(\lambda, u))-D_{1} P^{*} T(\lambda, u+f(\lambda, u))\right] .
$$

A computation shows that for $\beta \in \mathbf{R}, D_{1}((I-\lambda L) v)(\beta)=-\beta L v$. Therefore, $\left\|D_{1} f(\lambda, u)\right\| \leq\left\|\left[D_{2} F((\lambda, u), f(\lambda, u))\right]^{-1}\right\|\left(\|L\|\|f(\lambda, u)\|+\left\|D_{1} P^{*} T(\lambda, u+f(\lambda, u))\right\|\right)$.

Statement (iii) of the lemma then follows from statement (i) and H4.

\subsection{For the remainder of this paper we assume}

H5. Either $X$ is a Hilbert space or $\operatorname{dim} N\left(I-\lambda_{0} L\right)<\infty$.

We find it convenient to introduce an inner product on $N\left(I-\lambda_{0} L\right)$. If $X$ is a Hilbert space take $[$,$] to be the inner product of X$. If $X$ is not a Hilbert space choose a basis $u_{1}, u_{2}, \cdots, u_{m}$ of $N\left(I-\lambda_{0} L\right)$ with $\left\|u_{i}\right\|=1$ and let [, ] be the inner product defined by

$$
[u, \hat{u}]=\left[a_{1} u_{1}+\cdots+a_{m} u_{m}, b_{1} u_{1}+\cdots+b_{m} u_{m}\right]=a_{1} b_{1}+\cdots+a_{m} b_{m} .
$$

It can be verified that $[$,$] is a continuously differentiable map and there exist$ constants $c_{1}, c_{2}>0$ such that $c_{1}\|u\|^{2} \leq[u, u] \leq c_{2}\|u\|^{2}$ for all $u \in N\left(I-\lambda_{0} L\right)$ (for $X$ a Hilbert space, $c_{1}=c_{2}=1$ ).

Suppose $(\lambda, u, y)$ is a solution of equation (1.6). Then taking inner products

$$
[(I-\lambda L) u-P T(\lambda, u+f(\lambda, u, y), y), u]=0 .
$$

Simplifying, we obtain

$$
\lambda=[u, u]\left[L u+\lambda^{-1} P T(\lambda, u+f(\lambda, u, y), y), u\right]^{-1}
$$

when $y=0$ this becomes

$$
\lambda=[u, u]\left[L u+\lambda^{-1} P T(\lambda, u+f(\lambda, u)), u\right]^{-1} .
$$

Hence every solution of equation (1.6) or (1.8) is also a solution of equation (1.10) or (1.11). The converse of course need not be true. We can show, however, 
that for each $u$ sufficiently near 0 , equation (1.11) always has a unique solution $\lambda$.

Theorem 1.7. There exist a neighborbood $B$ of 0 in $N\left(I-\lambda_{0} L\right)$, an interval $A$ about $\lambda_{0}$ and a unique continuous function $\hat{\lambda}(u)$ mapping $B$ into $A$ such that the following are true:

(i) For $u \in B, \lambda=\hat{\lambda}(u)$ is the unique solution of equation (1.11) and $\hat{\lambda}(0)=\lambda_{D}$.

(ii) If $\lambda \in A$ and $u \in B$ satisfy equation (1.11) then $\lambda=\hat{\lambda}(u)$.

(iii) If $T \in C^{n}$ or is real analytic then in the deleted neighborbood of 0 , $\hat{\lambda}(u) \in C^{n}$ or is real analytic.

Proof. Let $G(\lambda, u)=[u, u]\left[L u+\lambda^{-1} P T(\lambda, u+f(\lambda, u)), u\right]^{-1}$. Then

$$
\begin{aligned}
G(\lambda, u) & =[u, u]\left\{\lambda_{0}^{-1}[u, u]+\lambda^{-1}[P T(\lambda, u+f(\lambda, u)), u]\right\}^{-1} \\
& =\left\{\lambda_{0}^{-1}+\lambda^{-1}[u, u]^{-1}[P T(\lambda, u+f(\lambda, u)), u]\right\}^{-1} .
\end{aligned}
$$

By our.previous discussion

$$
\left|[u, u]^{-1}[P T(\lambda, u+f(\lambda, u)), u]\right| \leq k_{1}\|u\|^{-1}\|P T(\lambda, u+f(\lambda, u))\| .
$$

Lemma 1.6 therefore, implies $G$ is continuous in a neighborhood of $\left(\lambda_{0}, 0\right)$ and $G(\lambda, 0)=\lambda_{0}$ for all $\lambda$ near $\lambda_{0}$. Clearly $D_{1} G$ exists and is continuous in an open set not containing points of the form $(\lambda, 0)$. We will show $D_{1} G$ exists and is continuous in an open neighborhood of $\left(\lambda_{0}, 0\right)$. For convenience let $\hat{T}(\lambda, u)=$ $[P T(\lambda, u+f(\lambda, u)), u] . \quad|\hat{T}(\lambda, u)| \leq k_{2}\|P T(\lambda, u+f(\lambda, u))\|\|u\|$ and so by Lemma 1.6

$$
|\hat{T}(\lambda, u)|=o\left(\|u\|^{2}\right)
$$

uniformly for $\lambda$ near $\lambda_{0}$. Furthermore, the same is true for $D_{1} \hat{T}$. For $\beta \in \mathbf{R}$, $D_{1} \hat{T}(\lambda, u)(\beta)$

$$
=\left[D_{1} P T(\lambda, u+f(\lambda, u))(\beta), u\right]+\left[D_{2} P T(\lambda, u+f(\lambda, u)) \circ D_{1} f(\lambda, u)(\beta), u\right] .
$$

Hence

$$
\left|D_{1} \hat{T}(\lambda, u)\right| \leq k_{2}\|u\|\left\{\left\|D_{1} P T(\lambda, u+f(\lambda, u))\right\|+\left\|D_{2} P T(\lambda, u+f(\lambda, u))\right\|\left\|D_{1} f(\lambda, u)\right\|\right\} .
$$

Thus by $\mathrm{H} 3, \mathrm{H} 4$ and Lemma 1.6

$$
\left|D_{1} \hat{T}(\lambda, u)\right|=\alpha\left(\|u\|^{2}\right)
$$

uniformly for $\lambda$ near $\lambda_{0}$.

Since $G(\lambda, 0)=\lambda_{0}$ for all $\lambda$ near $\lambda_{0}, D_{1} G(\lambda, 0) \equiv 0$ for $\lambda$ near $\lambda_{0}$. A computation shows that

$$
D_{1} G(\lambda, u)=-(G(\lambda, u))^{2}[u, u]^{-1}\left(-\lambda^{-2} \hat{T}(\lambda, u)+\lambda^{-1} D_{1} \hat{T}(\lambda, u)\right) .
$$

Therefore

$$
\left|D_{1} G(\lambda, u)\right| \leq k_{3}(G(\lambda, u))^{2}\|u\|^{-2}\left(|\lambda|^{-2}|\hat{T}(\lambda, u)|+|\lambda|^{-1}\left|D_{1} \hat{T}(\lambda, u)\right|\right) .
$$

By continuity $(G(\lambda, u))^{2}$ is uniformly bounded in some neighborhood of $\left(\lambda_{0}, 0\right)$. Therefore, by equations (1.12) and (1.13), $D_{1} G(\lambda, u) \rightarrow 0$ uniformly for $\lambda$ as 
$u \rightarrow 0$. Thus $D_{1} G$ is continuous in a neighborhood of $\left(\lambda_{0}, 0\right)$.

Let $F(\lambda, u)=G(\lambda, u)-\lambda . F$ and $D_{1} F$ are continuous. $F\left(\lambda_{0}, 0\right)=0$, $D_{1} F\left(\lambda_{0}, 0\right)=-1$ and $D_{1} F$ is a linear homeomorphism for $(\lambda, u)$ in a neighborhood of $\left(\lambda_{0}, 0\right)$. The results then follow by the implicit function theorem. (Conclusion (iii) follows when the implicit function theorem is applied to neighborhoods of points $(\lambda, u), u \neq 0$, near $\left(\lambda_{0}, 0\right)$.)

Theorem 1.7 simplifies the problem of finding solutions of equation (1.7). It is clear that if $u$ near 0 is a solution of

$$
(I-\hat{\lambda}(u) L) u-P T(\hat{\lambda}(u), u+f(\hat{\lambda}(u), u))=0
$$

then $(\lambda, u+f(\lambda, u))$ satisfies the homogeneous equation (1.7) where $\lambda=\hat{\lambda}(u)$.

Theorem 1.8. If for $\lambda \in \mathbf{R}, u$ is a solution of sufficiently small norm of either

$$
(I-\lambda L) u-P T(\hat{\lambda}(u), u+f(\hat{\lambda}(u), u))=0
$$

or

$$
(I-\lambda L) u-\lambda P T(u+f(\hat{\lambda}(u), u))=0
$$

(when $\left.T\left(\lambda_{j} x\right)=\lambda T(x)\right)$ then $\hat{\lambda}(u)=\lambda$ and $(\lambda, u+f(\lambda, u)$ ) is a solution of equation (1.7).

Proof. We prove the theorem for equation (1.14). If $(\lambda, u)$ satisfies equation (1.14) $u=\lambda L u+P T(\beta, u+f(\beta, u)), \beta=\hat{\lambda}(u)$.

Since $L u=\lambda_{0}^{-1} u$ there is a $\gamma$ such that $\gamma u=\beta L u+P T(\beta, u+f(\beta, u))$. Then $\gamma / \beta=[u, u]^{-1}\left[L u+\beta^{-1} P T(\beta, u+f(\beta, u)), u\right]=\hat{\lambda}(u)^{-1}=1 / \beta$. Hence $\gamma=1$ and the result follows. The proof for equation (1.15) is similar.

Corollary 1.9. If for some $\beta \in \mathbf{R}$ and $u$ in a small neighborbood of 0 , $\operatorname{PT}(\hat{\lambda}(u), u+f(\hat{\lambda}(u), u))=\beta u$ then $(\hat{\lambda}, u+f(\hat{\lambda}, u))$ is a solution of equation (1.7) where $\hat{\lambda}=\hat{\lambda}(u)$.

Proof. Since $L u=\lambda_{0}^{-1} u$ there exists a $\beta_{1}$ such that $\left(I-\beta_{1} L\right) u-$ $\operatorname{PT}(\hat{\lambda}, u+f(\hat{\lambda}, u))=0$. Hence by our previous theorem the result follows.

Theorem 1.10 (uniqueness). Suppose $(\lambda, x)$ and $\left(\lambda^{\prime}, x^{\prime}\right)$ are solutions of equation (1.7) near $\left(\lambda_{0}, 0\right)$ and $P x=P x^{\prime}$. Then $(\lambda, x)=\left(\lambda^{\prime}, x^{\prime}\right)$.

Proof. By Corollary $1.3 x=u+f(\lambda, u)$ and $x^{\prime}=u+f\left(\lambda^{\prime}, u\right)$. As we have seen in our discussion preceding Theorem $1.7,(\lambda, u)$ and $\left(\lambda^{\prime}, u\right)$ are solutions of equation (1.11). But by Theorem 1.7, $\lambda=\lambda^{\prime}$. Hence $(\lambda, x)^{\circ}=\left(\lambda^{\prime}, x^{\prime}\right)$.

\section{PART II. BIFURCATION FROM A SIMPLE EIGENVALUE}

2.1. Let $X$ and $Y$ be Banach spaces. In this section we prove the existence of nontrivial solutions of the nonlinear operator equation

$$
(I-\lambda L) x-T(\lambda, x, 0)=0
$$


near $\left(\lambda_{0}, 0\right)$ in $\mathbf{R} \times X . \lambda_{0}$ is a simple eigenvalue of the bounded linear operator $L$, and $T$ is a continuous map of a neighborhood of $\left(\lambda_{0}, 0,0\right)$ in $\mathbf{R} \times X \times Y$ into $X$. We recall that an eigenvalue $\lambda$ is called simple if all the solutions of the equations $(I-\lambda L)^{n} x=0 \quad(n=1,2, \ldots)$ are simultaneously solutions of the equation $(I-\lambda L) x=0$ and $\operatorname{dim} N(I-\lambda L)=1,[5]$.

We assume $I-\lambda_{0} L$ is a Fredholm operator of index 0 and $T$ satisfies conditions H2-H4 of Part I. Under these hypotheses the reductions of Part I apply to our equation.

To simplify the notation we express equation (2.1) as

$$
(I-\lambda L) x-T(\lambda, x)=0 .
$$

For our work in this section and in $\$ 2.2$ let $u_{0}$ be a fixed normalized vector in $N\left(I-\lambda_{0} L\right)$.

Theorem 2.1. Suppose $\lambda_{0}$ is a simple eigenvalue of $L, I-\lambda_{0} L$ is a Fredbolm operator of index 0 , and $T$ satisfies conditions $\mathrm{H} 2-\mathrm{H} 4$ of Part $\mathrm{I}$.

(i) $\lambda_{0}$ is a bifurcation point of equation (2.2) with respect to 0 .

(ii) There exists an interval $A$ about 0 , sucb that for each nonzero $\epsilon \in A$ equation (2.2) has a unique nontrivial solution of the form

$$
x=x(\epsilon)=\epsilon u_{0}+v(\epsilon), \quad \lambda=\lambda(\epsilon),
$$

where $v(\epsilon) \epsilon R\left(I-\lambda_{0} L\right), v(\epsilon)$ and $\lambda(\epsilon)$ are continuous, $\|v(\epsilon)\|=o(|\epsilon|)$ and $\lambda(0)=\lambda_{0}$.

(iii) Moreover, every solution near $\left(\lambda_{0}, 0\right)$ of equation (2.2) is of the form given in statement (ii).

(iv) If $T(\lambda, x)=\lambda T(x)$ then

$$
\lambda(\epsilon)=\lambda_{0}\left\{1+\lambda_{0} \epsilon^{-1}\left[P T(x(\epsilon)), u_{0}\right]\right\}^{-1} .
$$

Proof. We have shown in Part I that if $(\lambda, u) \in \mathbf{R} \times N\left(I-\lambda_{0} L\right)$ is a solution of equation (1.8) then $(\lambda, u)$ is also a solution of equation (1.11). When $\lambda_{0}$ is a simple eigenvalue the converse is also true. Suppose $(\lambda, u)=\left(\lambda, \epsilon u_{0}\right)$ is a solution of the equation (1.11). Then $[(I-\lambda L) u-P T(\lambda, u+f(\lambda, u)), u]=0$. Dividing by $\epsilon$ we get

$$
(I-\lambda L) u-P T(\lambda, u+f(\lambda, u))=\left[(I-\lambda L) u-P T(\lambda, u+f(\lambda, u)), u_{0}\right] u_{0}=0 .
$$

Consequently, $(\lambda, u)=\left(\lambda, \epsilon u_{0}\right)$ is a solution of equation (1.8). By Theorem 1.7 there exists a neighborhood $A$ of 0 in $R$ such that for each $\epsilon \in A,\left(\hat{\lambda}\left(\epsilon u_{0}\right), \epsilon u_{0}\right)$ is the unique solution of equation (1.11). Thus, by Corollary 1.3, for each nonzero $\epsilon \epsilon A,(\lambda(\epsilon), x(\epsilon))$ is the unique nontrivial solution of equation (2.2), where

$$
x(\epsilon)=\epsilon u_{0}+v(\epsilon)=\epsilon u_{0}+f\left(\lambda(\epsilon), \epsilon u_{0}\right)
$$

and

$$
\lambda(\epsilon)=\hat{\lambda}\left(\epsilon u_{0}\right)
$$


Since $f(\lambda, u) \in R\left(I-\lambda_{0} L\right), v(\epsilon) \in R\left(I-\lambda_{0} L\right)$. The continuity of $x(\epsilon)$ and $\lambda(\epsilon)$ is a consequence of their definitions and of Theorems 1.2 and 1.7. Moreover, since $\left(\hat{\lambda}\left(\epsilon \cdot u_{0}\right), \epsilon u_{0}\right)$ is the unique solution of equation $(1.11), \lambda(0)=\hat{\lambda}(0)=\lambda_{0}$. The equation $\|v(\epsilon)\|=o(|\epsilon|)$ is a consequence of Lemma 1.6. Therefore, we can also conclude that $\lambda_{0}$ is a bifurcation point of equation (2.2) with respect to 0 .

Statement (iii) is seen to be a consequence of the uniqueness of $f(\lambda, u)$ and $\hat{\lambda}(u)$.

To verify statement (iv), suppose $T(\lambda, x)=\lambda T(x)$. Then, by Theorem 1.7 , equation (1.11) becomes

$$
\hat{\lambda}\left(\epsilon u_{0}\right)=\lambda_{0}\left\{1+\lambda_{0} \epsilon^{-1}\left[P T\left(\epsilon u_{0}+f\left(\hat{\lambda}\left(\epsilon u_{0}\right), \epsilon u_{0}\right)\right), u_{0}\right]\right\}^{-1}
$$

which gives us equation (2.3).

The functions $v(\epsilon)$ and $\lambda(\epsilon)$ of Theorem 2.1 are defined in terms of $f(\lambda, u)$ and $\hat{\lambda}(u)$. We therefore, reach several conclusions about the differentiability of $v(\epsilon)$ and $\lambda(\epsilon)$.

Theorem 2.2. If in addition to the bypotbeses of Theorem 2.1, TE $C^{n}$, then the following are true:

(i) $v(\epsilon) \in C^{n-1}(A)$ and $\lambda(\epsilon) \in C^{n-1}(A)$.

(ii) $v(\epsilon) \in C^{n}(A-\{0\})$ and $\lambda(\epsilon) \in C^{n}(A-\{0\})$.

(iii) If $n \geq 2$ then $\left|\lambda(\epsilon)-\lambda_{0}\right|=O(|\epsilon|)$.

Proof. Equation (1.11) is equivalent to

$$
\lambda=\left\{\lambda_{0}^{-1}+(\lambda[u, u])^{-1}[P T(\lambda, u+f(\lambda, u)), u]\right\}^{-1} .
$$

Hence $\lambda=\left(\lambda_{0}^{-1}+\epsilon^{-1} E(\lambda, \epsilon)\right)^{-1}$ where $E(\lambda, \epsilon)=\lambda^{-1}\left[P T\left(\lambda, \epsilon u_{0}+f\left(\lambda, \epsilon u_{0}\right)\right), u_{0}\right]$. $E$ is a function of two real variables. $E(\lambda, 0)=0$ for all $\lambda$ near $\lambda_{0}$ and $E \in C^{n}$. By a real variables argument $\epsilon^{-1} E(\lambda, \epsilon) \epsilon C^{n-1}$. Since $\hat{\lambda}\left(\epsilon u_{0}\right)$ is defined via the implicit function theorem, $\hat{\lambda}\left(\epsilon u_{0}\right) \epsilon C^{n-1}$ in a neighborhood of 0 . Hence by equations (2.4) and (2.5) and Theorems 1.2 and 1.7 statements (i) and (ii) follow.

If $n \geq 2$ then by statement (i), $\lambda(\epsilon) \in C^{1}$. Therefore, $\lambda(\epsilon)$ must satisfy a uniform Lipschitz condition near 0 and statement (iii) follows.

Theorem 2.3. If in addition to the bypotheses of Theorem 2.1, $T$ is also real analytic then so are $\nu(\epsilon)$ and $\lambda(\epsilon)$. This implies that all solutions of equation (2.2) near $\left(\lambda_{0}, 0\right)$ are of the form

$$
\begin{aligned}
& x=\epsilon u_{0}+\sum_{n=2}^{\infty} \epsilon^{n} v_{n}, \\
& \lambda=\lambda_{0}+\sum_{n=1}^{\infty} \lambda_{n} \epsilon^{n} .
\end{aligned}
$$


Proof. Let $E(\lambda, \epsilon)$ be as defined in Theorem 2.2. Then, since $E$ is real analytic

$$
E(\lambda, \epsilon)=\sum_{n=0}^{\infty} f_{n}(\lambda) \epsilon^{n}
$$

where $f_{n}$ is real analytic for $n=0,1,2, \ldots$. However, since $E(\lambda, 0)=0$ and $D_{2} E(\lambda, 0)=0$ for $\lambda$ near $\lambda_{0}, f_{0}(\lambda) \equiv 0$ and $f_{1}(\lambda) \equiv 0$. Therefore, $\epsilon^{-1} E(\lambda, \epsilon)$ is real analytic. The function $\hat{\lambda}\left(\epsilon u_{0}\right)$ is defined by an application of the implicit function theorem and is therefore real analytic. Hence $\lambda(\epsilon)$ is real analytic. In addition, since the composition of analytic functions is real analytic, it follows from equation (2.4) that $v(\epsilon)$ is also real analytic.

Many problems in mathematics require that for each $\lambda$ near $\dot{\lambda}_{0}$ one determine the number of solutions of equation (2.2). Since for each $\epsilon$ near 0 there is a unique eigenvector $x(\epsilon)$, to arrive at the number of eigenvectors corresponding to an eigenvalue $\lambda$ one need only compute the number of distinct $\epsilon$ such that $\lambda=\lambda(\epsilon)$. In general each eigenvalue may have many eigenvectors (see Example 3 in Part IV). If, however, $T$ is real analytic then unless $\lambda_{0}$ is the sole eigenvalue each eigenvalue has at most two eigenvectors.

Theorem 2.4. Suppose $L$ and $T$ are as in Theorem 2.3.

(i) If $\lambda(\epsilon) \equiv \lambda_{0}$ then $\lambda_{0}$ is the only eigenvalue of equation (2.2) for small $\|x\|$.

(ii) If $\lambda(\epsilon) \equiv \lambda_{0}$ there is a smallest positive integer $N$ such that the coefficient $\lambda_{N}$ in the power series (2.7) is nonvanishing. In addition, equation (2.2) does not bave nonzero solutions of small norm for $\lambda=\lambda_{0}$.

(iii) If $N$ is odd, for each $\lambda$ near $\lambda_{0}$, equation (2.2) bas exactly one nontrivial solution $\hat{x}(\lambda)$ near 0 . The function $\hat{x}(\lambda)$ is continuous for $\lambda \epsilon$ $\left(\lambda_{0}-\delta_{1}, \lambda_{0}+\delta_{2}\right)$. Moreover, if $N=1, \hat{x}(\lambda)$ is real analytic in $\left(\lambda_{0}-\delta_{1}, \lambda_{0}+\delta_{2}\right)$, and if $N>1, \hat{x}(\lambda)$ is real analytic in $\left(\lambda_{0}-\delta_{1}, \lambda_{0}\right)$ and in $\left(\lambda_{0}, \lambda_{0}+\delta_{2}\right)$.

(iv) If $N$ is even and $\lambda_{N}>0\left(\lambda_{N}<0\right)$, for each $\lambda \in\left(\lambda_{0}, \lambda_{0}+\delta\right)\left(\lambda \in\left(\lambda_{0}-\right.\right.$ $\left.\delta, \lambda_{0}\right)$ ) equation (2.2) has exactly two nontrivial solutions $\hat{x}_{1}(\lambda)$ and $\hat{x}_{2}(\lambda)$ near zero and there are no solutions of small norm for $\lambda \in\left(\lambda_{0}-\delta, \lambda_{0}\right)\left(\lambda \in\left(\lambda_{0}, \lambda_{0}+\delta\right)\right)$. The functions $\hat{x}_{1}(\lambda)$ and $\hat{x}_{2}(\lambda)$ are continuous for $\lambda \in\left[\lambda_{0}, \lambda_{0}+\delta\right)\left(\lambda \in\left(\lambda_{0}-\delta, \lambda_{0}\right]\right)$. In addition, $\hat{x}_{1}(\lambda)$ and $\hat{x}_{2}(\lambda)$ are real analytic in $\left(\lambda_{0}, \lambda_{0}+\delta\right)\left(\left(\lambda_{0}-\delta, \lambda_{0}\right)\right)$.

Proof. By virtue of Theorem 2.3 and the analyticity of $\lambda(\epsilon)$, statements (i) and (ii) are obvious.

The rest of the theorem is proved by considering the derivative of $\lambda(\epsilon)$ :

$$
\lambda^{\prime}(\epsilon)=\sum_{n=N}^{\infty} \lambda_{n} n \epsilon^{n-1} .
$$

Therefore $\lambda^{\prime}(\epsilon)=\lambda_{N} N \epsilon^{N-1}+b(\epsilon)$, where $|b(\epsilon)|=o\left(|\epsilon|^{N-1}\right)$.

Suppose $N$ is odd. Then $N-1$ is even and for $\epsilon \neq 0, \lambda_{N} \epsilon^{N-1}$ has 
constant sign. Since $|b(\epsilon)|=o\left(|\epsilon|^{N-1}\right)$, sign $\lambda^{\prime}(\epsilon)=\operatorname{sign} \lambda_{N} N \epsilon^{N-1}$ for $\epsilon$ near 0 . Therefore, $\lambda(\epsilon)$ is a monotone function on an interval $\left(-\epsilon_{0}, \epsilon_{0}\right)$. The range of $\lambda(\epsilon)$ must then be an interval $\left(\lambda_{0}-\delta_{1}, \lambda_{0}+\delta_{2}\right)$.

If $\lambda_{1} \neq 0$ then $\lambda^{\prime}(\epsilon) \neq 0$ for all $\epsilon \epsilon\left(-\epsilon_{0}, \epsilon_{0}\right)$. By the implicit function theorem $\lambda(\epsilon)$ has a real analytic inverse $i(\lambda)$. The map $\hat{x}(\lambda)=x(i(\lambda))$ for $\lambda \epsilon$ $\left(\lambda_{0}-\delta_{1}, \lambda_{0}+\delta_{2}\right)$, gives us statement (iii) for $N=1$.

If $N>1$ and odd, $\lambda^{\prime}(\epsilon) \neq 0$ for $\epsilon \neq 0, \epsilon \in\left(-\epsilon_{0}, \epsilon_{0}\right)$. For each $\delta>0$ the function $\lambda(\epsilon)$ restricted to $\left(-\epsilon_{0},-\delta\right.$ ] has a real analytic inverse and the function $\lambda(\epsilon)$ restricted to $\left[\delta, \epsilon_{0}\right)$ has a real analytic inverse. Thus there exists a continuous inverse $i(\lambda)$ of $\lambda(\epsilon)$ mapping $\left(\lambda_{0}-\delta_{1}, \lambda_{0}+\delta_{2}\right)$ into $\left(-\epsilon_{0}, \epsilon_{0}\right)$. Furthermore, $i(\lambda)$ is real analytic in $\left(\lambda_{0}-\delta_{1}, \lambda_{0}\right)$ and in $\left(\lambda_{0}, \lambda_{0}+\delta_{2}\right)$. The map $\hat{x}(\lambda)=$ $x(i(\lambda)), \lambda \in\left(\lambda_{0}-\delta_{1}, \lambda_{0}+\delta_{2}\right)$ gives us statement (iii).

Suppose $N$ is even. We assume $\lambda_{N}>0$. The case of $\lambda_{N}<0$ is handled analogously. $N-1$ is odd, which means $\lambda_{N} N \epsilon^{N-1}<0$ for $\epsilon<0$ and $\lambda_{N} N \epsilon^{N-1}>0$ for $\epsilon>0$. Hence for $\epsilon<0$ and near $0, \lambda^{\prime}(\epsilon)<0$ and $\lambda(\epsilon)$ is thus a decreasing function. On the other hand, for $\epsilon>0$ and near $0, \lambda^{\prime}(\epsilon)>0$, and $\lambda(\epsilon)$ is an increasing function. Therefore $\lambda_{0} \leq \lambda(\epsilon)$ for all $\epsilon$ near 0 and the range of $\lambda(\epsilon)$ is a half open interval $\left[\lambda_{0}, \lambda_{0}+\delta_{1}\right)$. This implies there are no solutions of small norm of equation (2.2) for $\lambda \epsilon\left(\lambda_{0}-\delta_{1}, \lambda_{0}\right)$. For each $\delta<0$ the function $\lambda(\epsilon)$ restricted to $\left(-\epsilon_{0}, \delta\right]$ has a real analytic inverse. As before, $\lambda(\epsilon)$ restricted to $\left(-\epsilon_{0}, 0\right]$, has a continuous inverse $i_{1}(\lambda)$ and $i_{1}(\lambda)$ is real analytic in $\left(\lambda_{0}, \lambda_{0}+\delta_{1}\right)$. Similarly, $\lambda(\epsilon)$ restricted to $\left[0, \epsilon_{0}\right)$ has a continuous inverse $i_{2}(\lambda)$ and $i_{2}(\lambda)$ is real analytic in $\left(\lambda_{0}, \lambda_{0}+\delta_{1}\right)$. The maps $\hat{x}_{1}(\lambda)=$ $x\left(i_{1}(\lambda)\right)$ and $\hat{x}_{2}(\lambda)=x\left(i_{2}(\lambda)\right)$ for $\lambda \in\left[\lambda_{0}, \lambda_{0}+\delta_{1}\right)$ give us statement (iv).

Theorem 2.4 implies the following:

Corollary 2.5. If $L$ and $T$ are as in Theorem 2.1 and $T$ is real analytic, then either $\lambda_{0}$ is the only eigenvalue of equation (2.2) for $x$ of small norm, or the eigenvalues of equation (2.2) near $\lambda_{0}$ have at most two nontrivial solutions of small norm.

In general, unless more is known about the operator $T, \lambda_{N}$ of Theorem 2.4 must be computed to determine if the solutions of equation (2.2) are as in statements (i), (iii) or (iv). The next two corollaries give conditions on $T$ which enable us to ascertain the nature of the solutions without explicitly evaluating $\lambda_{N}$.

Corollary 2.6. If $T$ is real analytic and odd (that is, $T(\lambda,-x)=-T(\lambda, x)$ ), then either $\lambda_{0}$ is the only eigenvalue near $\lambda_{0}$ of equation (2.2) for $x$ of small norm, or there exist just two continuous solution functions $\hat{x}_{1}(\lambda)$ and $\hat{x}_{2}(\lambda)$ of equation (2.2) for $\lambda \epsilon\left[\lambda_{0}, \lambda_{0}+\delta\right)$ or $\lambda \epsilon\left(\lambda_{0}-\delta, \lambda_{0}\right)$ and there are no small solutions for $\lambda \in\left(\lambda_{0}-\delta, \lambda_{0}\right]$ or $\lambda \in\left[\lambda_{0}, \lambda_{0}+\delta\right)$. 
Proof. $T$ is odd; therefore if $(\lambda, x)$ is a solution of equation (2.2) then so is $(\lambda,-x)$. Hence there exist at least two distinct solutions for each eigenvalue. Statement (iii) of Theorem 2.4, which implies the existence of just one solution for each $\lambda$ obviously does not obtain here. The solutions must therefore be as in statement (i) or (iv) of Theorem 2.4. Hence the result.

Corollary 2.7. Suppose $T$ is real analytic and $T(\lambda, x)=b(\lambda) A_{N}(x)+R(\lambda, x)$ where $b(\lambda)$ is a real-valued analytic function, $A_{N}(x)=a_{N}\left(x^{N}\right), a_{N}$ a symmetric $N$-linear form, and $\|R(\lambda, x)\|=o\left(\|x\|^{N}\right)$ for each $\lambda$ near $\lambda_{0}$. If in addition $\left[\mathrm{Pb}\left(\lambda_{0}\right) A_{N}(u), u\right] \neq 0$ for $u \in N\left(I-\lambda_{0} L\right), u \neq 0$, then the following are true:

(i) If $N$ is even there exists exactly one nontrivial continu@us solution function $\hat{x}(\lambda)$ of equation (2.2) depending on $\lambda$ for $\lambda \in\left(\lambda_{0}-\delta_{1}, \lambda_{0}+\delta_{2}\right)$.

(ii) If $N$ is odd and $\lambda_{0}\left[P b\left(\lambda_{0}\right) A_{N}(u), u\right]<0\left(\lambda_{0}\left[P b\left(\lambda_{0}\right) A_{N}(u), u\right]>0\right)$ for $u$ $\epsilon N\left(I-\lambda_{0} L\right), u \neq 0$, then there exist exactly two distinct nontrivial continuous solution functions. The functions, $\hat{x}_{1}(\lambda)$ and $\hat{x}_{2}(\lambda)$, depend on $\lambda$ for $\lambda \epsilon$ $\left[\lambda_{0}, \lambda_{0}+\delta\right)\left(\lambda \in\left(\lambda_{0}-\delta, \lambda_{0}\right]\right)$ and there are no solutions of small norm of equation (2.2) for $\lambda \in\left(\lambda_{0}-\delta, \lambda_{0}\right)\left(\lambda \in\left(\lambda_{0}, \lambda_{0}+\delta\right)\right)$.

Proof. We prove this the orem by finding $\lambda_{N}$ of Theorem 2.4. Using the definition of $\lambda(\epsilon)$ and $\hat{\lambda}\left(\epsilon u_{0}\right)$ and equation (1.11) we get the equation

$$
\begin{aligned}
\lambda(\epsilon) & =\hat{\lambda}\left(\epsilon u_{0}\right)=\left\{\lambda_{0}^{-1}+(\lambda \epsilon)^{-1} S(\lambda, \epsilon)\right\}^{-1}=\lambda_{0}\left\{1+\lambda_{0}(\lambda \epsilon)^{-1} S(\lambda, \epsilon)\right\}^{-1} \\
& =\lambda_{0}-\lambda_{0}^{2}(\lambda \epsilon)^{-1} S(\lambda, \epsilon)+\lambda_{0} \sum_{n=2}^{\infty}(-1)^{n}\left\{\lambda_{0}(\lambda \epsilon)^{-1} S(\lambda, \epsilon)\right\}^{n}
\end{aligned}
$$

where

$$
S(\lambda, \epsilon)=\left[P T\left(\lambda, \epsilon u_{0}+f\left(\lambda(\epsilon), \epsilon u_{0}\right)\right), u_{0}\right]
$$

For $u \in N\left(I-\lambda_{0} L\right)$,

$$
\begin{aligned}
P A_{N}(u+f(\lambda, u)) & =\sum_{i+j=N} \frac{N !}{i ! j !} P a_{N}\left(u^{i}, f(\lambda, u)\right) \\
& =P A_{N}(u)+\sum_{i+j=N ; j \neq 0} \frac{N !}{i ! j !} P a_{N}\left(u^{i}, f^{j}(\lambda, u)\right) .
\end{aligned}
$$

By Lemma 1.6 we have

$$
\left\|\sum_{i+j=N ; j \neq 0} \frac{N !}{i ! j !} P a_{N}\left(u^{i}, f^{j}(\lambda, u)\right)\right\| \leq \sum_{i+j=N ; j \neq 0} c_{i j}\|u\|^{i}\|f(\lambda, u)\|^{j}=o\left(\|u\|^{N}\right)
$$

uniformly for $\lambda$ near $\lambda_{0}$. Therefore, by equation (2.9) $S(\lambda, \epsilon)=b(\lambda) a \epsilon^{N}+$ $E(\lambda, \epsilon)$ where $a=\left[P A_{N}\left(u_{0}\right), u_{0}\right]$ and $|E(\lambda, \epsilon)|=o\left(|\epsilon|^{N}\right)$ for each $\lambda$. Since $S$ is real analytic 


$$
S(\lambda, \epsilon)=b(\lambda) a \epsilon^{N}+\sum_{n=N+1}^{\infty} f_{n}(\lambda) \epsilon^{n}
$$

where $f_{n}(\lambda)$ is analytic for $n=N+1, N+2, \cdots$. Now since $b(\lambda)$ and $\lambda(\epsilon)$ are analytic,

$$
b(\lambda)=\beta_{0}+\sum_{n=1}^{\infty} \beta_{n}\left(\lambda-\lambda_{0}\right)^{n}, \quad \beta_{0} \neq 0,
$$

and

$$
b(\lambda(\epsilon))=\beta_{0}+\sum_{n=1}^{\infty} \beta_{n}\left(\sum_{i=1}^{\infty} \lambda_{i} \epsilon^{i}\right)^{n}=\beta_{0}+\sum_{n=1}^{\infty} b_{n} \epsilon^{n} .
$$

Combining equations (2.10) and (2.11) we get

$$
\hat{S}(\epsilon)=S(\lambda(\epsilon), \epsilon)=\beta_{0} a \epsilon^{N}+\sum_{n=N+1}^{\infty} c_{n} \epsilon^{n} .
$$

Since $\lambda(\epsilon)$ is real analytic and $\lambda(0)=\lambda_{0}$ we have

$$
\lambda(\epsilon)^{-1}=\frac{1}{\lambda_{0}}+\sum_{n=1}^{\infty} d_{n} \epsilon^{n} .
$$

From equations (2.12), (2.8) and (2.13) we get

$$
\lambda(\epsilon)=\lambda_{0}-\lambda_{0} \beta_{0} a \epsilon^{N-1}+\sum_{n=N}^{\infty} e_{n} \epsilon^{n} .
$$

By hypothesis, $-\lambda_{0} \beta_{0} a=-\lambda_{0}\left[P b\left(\lambda_{0}\right) A_{N}\left(u_{0}\right), u_{0}\right] \neq 0$. Hence if $N$ is even, then $N-1$ is odd and statement (i) follows from Theorem 2.4. Similarly, if $N$ is odd, $N-1$ is even and statement (ii) follows from Theorem 2.4.

2.2. Let $X$ and $Y$ be Banach spaces. In this section we solve the bifurcation problem for the nonhomogene ous equation

$$
(I-\lambda L) x-T(\lambda, x, y)=0
$$

near $\left(\lambda_{0}, 0,0\right)$ in $\mathbf{R} \times X \times Y$, where $\lambda_{0}$ is a simple eigenvalue of $L$, and $L$ and $T$ are as in $\$ 2.1$.

For $\epsilon_{0}>0$ we let $B_{\epsilon_{0}}=\left\{(\epsilon, 0) \in \mathbf{R} \times Y|0<| \epsilon \mid<\epsilon_{0}\right\}$.

Theorem 2.8. Suppose $\lambda_{0}$ is a simple eigenvalue of $L, I-\lambda_{0} L$ is a Fredbolm operator of index 0 , and $T$ satisfies conditions $\mathrm{H} 2-\mathrm{H} 4$ of Part $\mathrm{I}$.

(i) There is a subset $B_{\epsilon_{0}}$ of $\mathbf{R} \times Y$, and an open set $A$ containing $B_{\epsilon_{0}}$, such that for each $(\epsilon, y) \in A$ equation (2.14) has a unique solution of the form

$$
\lambda=\lambda(\epsilon, y), \quad x=x(\epsilon, y)=\epsilon u_{0}+v(\epsilon, y), \quad y=y,
$$

where $v(\epsilon, y) \in R\left(I-\lambda_{0} L\right)$ and $\lambda(\epsilon, y)$ and $v(\epsilon, y)$ are continuous. In addition, 
$x(\epsilon, 0)=x(\epsilon)$ and $\lambda(\epsilon, 0)=\lambda(\epsilon)$, where $x(\epsilon)$ and $\lambda(\epsilon)$ are the unique functions of Theorem 2.1 .

(ii) If a solution $(\lambda, x, 0)$ of equation (2.1) is sufficiently near $\left(\lambda_{0}, 0,0\right)$ then every solution of equation (2.14) in a small neighborbood of $(\lambda, x, 0)$ is of the form shown in statement (i).

(iii) If $T \in C^{2}$ then $\|v(\epsilon, y)\|=o\left(\epsilon^{2}+\|y\|\right)$, and if $\|y\| \leq \epsilon^{2}$ then $\mid \lambda(\epsilon, y)$ $\lambda_{0} \mid=O(|\epsilon|)$.

(iv) If $T \in C^{n}$ then so are $\lambda(\epsilon, y)$ and $v(\epsilon, y)$.

(v) If $T$ is real analytic then so are $\lambda(\epsilon, y)$ and $\nu(\epsilon, y)$.

Proof. An argument similar to the one employed in the proof of Theorem 2.1 will show $(\lambda, u, y)$ is a solution of equation (1.6) if and only if- $(\lambda, u, y)$ is a solution of equation (1.10). We will therefore find solutions of equation (1.10).

Let

$$
F(\lambda,(u, y))=[u, u]\left[L u+\lambda^{-1} P T(\lambda, u+f(\lambda, u, y), y), u\right]^{-1}-\lambda
$$

In the course of proving Theorem 1.7 we showed $D_{1} F\left(\lambda_{0},(0,0)\right)$ is a linear homeomorphism. There is an open interval $A_{\lambda_{0}}$ about $\lambda_{0}$ and a neighborhood $A_{0}$ of 0 in $N\left(I-\lambda_{0} L\right)$ such that for $(\lambda, u) \in A_{\lambda_{0}} \times A_{0}, D_{1} F(\lambda,(u, 0))$ is a linear homeomorphism and $A_{0}$ is a subset of the domain of $\hat{\lambda}(u)$.

Now let $\hat{u} \in A_{0}, \hat{u} \neq 0$. There is a unique $\hat{\lambda}=\hat{\lambda}(\hat{u})$ such that $(\hat{\lambda}, \hat{u}, 0)$ satisfies equation (1.11). Therefore

$$
\left[L \hat{u}+\hat{\lambda}^{-1} P T(\hat{\lambda}, \hat{u}+f(\hat{\lambda}, \hat{u}, 0)), \hat{u}\right] \neq 0 .
$$

Thus there is a neighborhood $B$ of $(\hat{\lambda}, \hat{u}, 0)$ in which $F$ is continuous, $F$ is in $C^{n}$ or is real analytic as $T$ is in $C^{n}$ or is real analytic, and $D_{1} F$ is a linear homeomorphism. The hypotheses of the implicit function theorem are satisfied. Thus there exist an open interval $N_{1}(\hat{u}) \subseteq A_{\lambda_{0}}$ about $\hat{\lambda}$, a neighborhood $N_{2}(\hat{u}) \subseteq A_{0}$ of $\hat{u}$, a sphere $N_{3}(\hat{u})$ of small radius about 0 in $Y$, where $N_{1}(\hat{u}) \times$ $N_{2}(\hat{u}) \times N_{3}(\hat{u}) \subseteq B$, and a continuous function $\hat{\lambda}(u, y)$ mapping $A(\hat{u})=N_{2}(\hat{u}) \times$ $N_{3}(\hat{u})$ into $N_{1}(\hat{\hat{u}})$ such that $(\hat{\lambda}(u, y), u, y)$ satisfies equation $(1.10)$ for all $(u, y) \in A(\hat{u})$. Moreover, if $(\lambda, u, y) \in N_{1}(\hat{u}) \times A(\hat{u})$ satisfies equation (1.10), then $\lambda=\hat{\lambda}(u, y)$. In addition, $\hat{\lambda}(u, v)$ is in $C^{n}$ or is real analytic as $T$ is in $C^{n}$ or is real analytic.

For each $\hat{u} \in A_{0}$ there exist a $\hat{\lambda}$, neighborhoods $N_{1}(\hat{u})$ and $A(\hat{u})$, and a function $\hat{\lambda}_{\hat{u}}(u, y)$ with the above properties. We must show that if $(u, y) \epsilon$ $A\left(u_{1}\right) \cap A\left(u_{2}\right)$ then $\hat{\lambda}_{u_{1}}(u, y)=\hat{\lambda}_{u_{2}}(u, y)$. By our previous arguments $\hat{\lambda}_{u_{1}}(u, 0)$ $=\hat{\lambda}_{u_{2}}(u, 0)$ (the unique solution of equation (1.11)). Let $t_{0}$ be the least upper bound for all $\hat{t}, 0 \leq \hat{t} \leq 1$, such that for $0 \leq t \leq \hat{t}, \hat{\lambda}_{u_{1}}(u, t y)=\hat{\lambda}_{u_{2}}(u, t y)$. By continuity, $\hat{\lambda}_{u_{1}}\left(u, t_{0} y\right)=\hat{\lambda}_{u_{2}}\left(u, t_{0} y\right)$. Since $D_{1} F$ e valuated at $\left(\hat{\lambda}_{u_{1}}\left(u, t_{0} y\right)\right.$, $\left.u, t_{0} y\right)$ is a linear homeomorphism, an application of the implicit function theorem 
to equation (2.15) shows that $\hat{\lambda}_{u}$ and $\hat{\lambda}_{u_{2}}$ can be extended uniquely for $t>t_{0}$. Therefore $t_{0}=1$ and $\hat{\lambda}_{u_{1}}(u, y)=\hat{\lambda}_{u_{2}}(u, y)$.

Let $\hat{A}=\bigcup_{u \neq 0} A(u)$ and let $A$ be the set of all $(\epsilon, y) \in R \times Y$ such that $\left(\epsilon u_{0}, y\right) \in \hat{A}$. If $(\epsilon, y) \in A$ then $\left(\epsilon u_{0}, y\right) \in A(\hat{u})$ for some $\hat{u}$ and we define $\lambda(\epsilon, y)=\hat{\lambda}_{\hat{u}}\left(\epsilon u_{0}, y\right)$. By the above argument $\lambda(\epsilon, y)$ is uniquely defined. For $(\epsilon, y) \in A,\left(\lambda(\epsilon, y), \epsilon u_{0}, y\right)$ is the unique solution of equation (1.10) and hence of (1.6). Thus, by Corollary 1.3, for each $(\epsilon, y) \in A,(\lambda(\epsilon, y), x(\epsilon, y), y)$ is the unique solution of equation (2.14) where

$$
x(\epsilon, y)=\epsilon u_{0}+v(\epsilon, y)=\epsilon u_{0}+f\left(\lambda(\epsilon, y), \epsilon u_{0}, y\right) .
$$

By our construction $\lambda(\epsilon, 0)=\lambda(\epsilon)$ and $x(\epsilon, 0)=x(\epsilon)$, where $\lambda(\epsilon)$ and $x(\epsilon)$ are the solutions of equation (2.1). Thus we have proved statement (i). In addition, by Theorem 1.2 and equation (2.16) $\lambda(\epsilon, y)$ and $x(\epsilon, y)$ are in $C^{n}$ or are real analytic as $T$ is in $C^{n}$ or is real analytic. Hence we have proved statements (iv) and (v).

Now we prove statement (ii). Suppose $(\lambda, x, y)$ is a solution of equation (2.14) in a small neighborhood of $(\hat{\lambda}, \hat{x}, 0)$, where $(\hat{\lambda}, \hat{x}, 0)$ is in turn a solution of equation (2.1) near $\left(\lambda_{0}, 0,0\right)$. By Corollary 1.3, $x=\epsilon u_{0}+f\left(\lambda, \epsilon u_{0}, y\right)$ and $\hat{x}=\hat{\epsilon} u_{0}+f\left(\hat{\lambda}, \hat{\epsilon} u_{0}, 0\right)$, and $\left(\lambda, \epsilon u_{0}, y\right)$ and $\left(\hat{\lambda}, \hat{\epsilon} u_{0}, 0\right)$ satisfy equation (1.6). By our previous arguments $\lambda=\hat{\lambda}_{\hat{\epsilon} u 0}\left(\epsilon u_{0}, y\right)=\lambda(\epsilon, y)$ and statement (ii) follows.

We proceed to prove statement (iii). Since $T(\lambda, x, y)$ is in $C^{2}$, by Theorem $1.2 f(\lambda, u, y)$ and $\operatorname{PT}(\lambda, u+f(\lambda, u, y), y)$ are also twice continuously differentiable. Moreover, $f(\lambda, 0,0)=0, D_{2} f(\lambda, 0,0)=0, \operatorname{PT}(\lambda, 0,0)=0$ and $D_{2} P T(\lambda, 0,0)=0$ for $\lambda$ near $\lambda_{0}$. Let $K(\lambda, u, y)$ be either $f(\lambda, u, y)$ or $P T(\lambda, u+f(\lambda, u, y), y)$. By continuity there is a neighborhood $S$ about $\left(\lambda_{0}, 0,0\right)$ such that $D K$ and $D^{2} K$ are uniformly bounded on $S$ and if $(\lambda, u, y) \in S$ then so is $(\lambda, t u, t y)$ for $0 \leq t \leq 1$. F or $(\lambda, u, y) \in S$, using the fact that for a continuously differentiable map $\hat{T}$ of an open subset $A$ of $X$ into $Y$, if $x+t y \in A$ for $0 \leq t \leq 1$, then

$$
\|\hat{T}(x+y)-\hat{T}(x)\| \leq \sup _{0 \leq t \leq 1}\|D \hat{T}(x+t y)(y)\| \leq\|y\| \sup _{0 \leq t \leq 1}\|D \hat{T}(x+t y)\|
$$

$[3$, p. 155], we have

$$
\begin{aligned}
& \|K(\lambda, u, y)\|=\|K(\lambda, u, y)-K(\lambda, 0,0)\| \\
& \quad \leq\|u\| \sup _{0 \leq t \leq 1}\left\|D_{2} K(\lambda, t u, t y)\right\|+\|y\| \sup _{0 \leq t \leq 1}\left\|D_{3} K(\lambda, t u, t y)\right\| .
\end{aligned}
$$

In addition,

$$
\begin{aligned}
& \left\|D_{2} K(\lambda, t u, t y)\right\|=\left\|D_{2} K(\lambda, t u, t y)-D_{2} K(\lambda, 0,0)\right\| \\
& \leq\|u\| \sup _{0 \leq s \leq 1}\left\|D_{2}^{2} K(\lambda, s u, s y)\right\|+\|y\| \sup _{0 \leq s \leq 1}\left\|D_{3} D_{2} K(\lambda, s u, s y)\right\| .
\end{aligned}
$$


Therefore, $\|K(\lambda, u, y)\|=O\left(\|u\|^{2}+\|u\| \cdot\|y\|+\|y\|\right)=O\left(\|u\|^{2}+\|y\|\right)$ independent of $\lambda$. Hence $\|v(\epsilon, y)\|=\left\|f\left(\lambda(\epsilon, y), \epsilon u_{0}, y\right)\right\|=O\left(\epsilon^{2}+\|y\|\right)$. If $\|y\| \leq \epsilon^{2}$ and $E(\lambda, \epsilon)=(\lambda \epsilon)^{-1}\left[P T\left(\lambda, \epsilon u_{0}+f\left(\lambda, \epsilon u_{0}, y\right), y\right), u_{0}\right]$ then by what we have just proved

$$
|E(\lambda, \epsilon)|=c|\lambda \epsilon|^{-1} O\left(\epsilon^{2}+\epsilon^{2}\right)=O(|\epsilon|) .
$$

Since $\lambda(\epsilon, y)$ is the unique solution of equation (1.10) for a given $\left(\epsilon u_{0}, y\right)$, if we let $\lambda=\lambda(\epsilon, y)$ we have

$$
\lambda(\epsilon, y)=\lambda_{0}\left(1+\lambda_{0} E(\lambda, \epsilon)\right)^{-1}=\lambda_{0}-\lambda_{0}^{2} E(\lambda, \epsilon)+\lambda_{0} \sum_{n=2}^{\infty}(-1)^{n}\left(\lambda_{0} E(\lambda, \epsilon)\right)^{n} .
$$

Thus $\left|\lambda(\epsilon, y)-\lambda_{0}\right|=O(|\epsilon|)$.

Remark. Theorem 2.8 shows the existence of solutions of equation (2.14) in a neighborhood of each nontrivial solution of equation (2.1). A stronger result is true for a neighborhood of a trivial solution of equation (2.1). Differentiating the left side of equation with respect to $x$, and evaluating at $(\lambda, 0,0)$, we get $I$ $\lambda L$. If $\lambda_{0}$ is an isolated element of the spectrum of $L$ and $\lambda \neq \lambda_{0}$, then $I-\lambda L$ is a linear homeomorphism of $X$ onto $X$. Hence by the implicit function theorem there exist a neighborhood $A$ of $(\lambda, 0)$ in $\mathbf{R} \times Y$ and a function $b(\lambda, y)$ such that for each $(\lambda, y) \in A,(\lambda, b(\lambda, y), y)$ is the unique solution of equation (2.14).

Suppose equation (2.14) has the special form

$$
(I-\lambda L) x-T\left(\lambda, x, \epsilon^{2} y\right)=0
$$

where $L$ and $T$ satisfy the hypotheses of Theorem $2.8, \lambda_{0}$ is a simple eigenvalue of $L$, and in addition, $D_{1} T(\lambda, x, y)=o\left(\|x\|+\|y\|^{1 / 2}\right)$ uniformly for $\lambda$ near $\lambda_{0}$.

Let $y$ be a fixed element of $Y$. We prove in our next theorem the existence of a solution set of equation (2.18) branching out from $\left(\lambda_{0}, 0,0\right)$ depending continuously on the real parameter $\epsilon$.

Theorem 2.9. Let $L$ and $T$ be as indicated above.

(i) Equation (2.18) has solutions of the form $\left(\lambda(\epsilon), x(\epsilon), \epsilon^{2} y\right)$ for $\epsilon$ near 0 , where $x(\epsilon)=\epsilon u_{0}+v(\epsilon), v(\epsilon) \in R\left(I-\lambda_{0} L\right) . \lambda(\epsilon)$ and $v(\epsilon)$ are continuous and $(\lambda(0)$, $x(0), 0)=\left(\lambda_{0}, 0,0\right)$.

(ii) If $T$ is in $C^{n}$ or is real analytic then $\lambda(\epsilon)$ and $\nu(\epsilon)$ are in $C^{n-1}$ or are real analytic.

Proof. Consider solutions of the equation

$$
(I-\lambda L) x-T(\lambda, x,[P x, P x] y)=0 .
$$

If $(\lambda, x)$ is a solution of equation (2.19) then $\left(\lambda, x, \epsilon^{2}\right)$ is a solution of equation (2.18), where $\epsilon^{2}=\left[\epsilon u_{0}, \epsilon u_{0}\right]=[P x, P x]$.

Let $\hat{T}(\lambda, x)=T(\lambda, x,[P x, P x] y)$ and consider the equation $(I-\lambda L) x-$ 
$\hat{T}(\lambda, x)=0$. Then since $L$ and $\hat{T}$ satisfy the hypotheses of Theorem 2.1 the results follow.

\section{PART III. BIFURCATION FROM MULTIPLE EIGENVALUES}

3.1. Consider the equation

$$
(I-\lambda L) x-T(\lambda, x)=0
$$

where $I-\lambda_{0} L$ is a Fredholm operator of index zero. $N\left(I-\lambda_{0} L\right) \cap R\left(I-\lambda_{0} L\right)=$ $\{0\}$ and $T$ is a nonlinear map of a neighborhood of $\left(\lambda_{0}, 0\right)$ into $X$ satisfying $\mathrm{H} 2-\mathrm{H} 4$.

Theorem 3.1. If the above bypotheses are satisfied and $\operatorname{dim} N\left(I-\lambda_{0} L\right)$ is odd then $\lambda_{0}$ is a bifurcation point of equation (3.1).

Proof. The theorem will be proved by the following lemma. If $H$ is a continuous map of $S_{r, n}$ into $\mathbf{R}^{n}$, where $\mathbf{R}^{n}$ is Euclidean $n$-space, $S_{r, n}$ is the surface of the sphere of radius $r$ in $\mathbf{R}^{n}$, and $n$ is odd, then there is some $x \in S_{r, n}$ and $\beta \in \mathbf{R}$ such that $H(x)=\beta x$. For a proof see $[2, \mathrm{p} .51]$.

By this lemma for each sufficiently small positive $r$ there is a $\beta_{r}$ and a $u_{r}$ such that $\left\|u_{r}\right\|=r$ and $\operatorname{PT}\left(\lambda_{r}, u_{r}+f\left(\lambda_{r}, u_{r}\right)\right)=\beta_{r} u_{r}$ where $\lambda_{r}=\hat{\lambda}\left(u_{r}\right)$. By Corollary $1.9\left(\lambda_{r}, u_{r}+f\left(\lambda_{r}, u_{r}\right)\right)$ is a solution of equation (3.1). Therefore, by the continuity of $\hat{\lambda}(u), \lambda_{0}$ is a point of bifurcation.

3.2. When $\operatorname{dim} N\left(I-\lambda_{0} L\right)$ is even solving the bifurcation problem of equation (3.1) becomes more difficult. There is a simple example in which $L$ is a selfadjoint operator in a Hilbert space, $\operatorname{dim} N\left(I-\lambda_{0} L\right)=2, L$ and $T$ satisfy $\mathrm{H} 1-\mathrm{H} 4$ and $T$ depends linearly on $\lambda$, yet there are no nontrivial solutions of equation (3.1) near $\left(\lambda_{0}, 0\right)$ (see [1, p. 121]). Thus in general when $\operatorname{dim} N\left(I-\lambda_{0} L\right)$ is even additional restrictions must be imposed on $T$ to guarantee the existence of solutions of small norm for $\lambda$ near $\lambda_{0}$.

Let us assume $\operatorname{dim} N\left(I-\lambda_{0} L\right)=2$.

Theorem 3.2. Suppose there exists a normalized basis $\left\{u_{1}, u_{2}\right\}$ of $N\left(I-\lambda_{0} L\right)$ sucb that $\left[P T\left(\lambda, b u_{2}+f\left(\lambda, b u_{2}\right)\right), u_{1}\right] \geq 0$ for small $|b|$ and all $\lambda$ near $\lambda_{0}$, where [, $]$ is defined as in $\$ 1.3$. Then $\lambda_{0}$ is a bifurcation point of equation (3.1) with respect to 0 .

Proof. PT, $f(\lambda, u)$ and $\hat{\lambda}(u)$ are cont inuous in $N\left(I-\lambda_{0} L\right)$ with norm \|\|$_{H}=$ $[,]^{1 / 2}$. For $x, y \in N\left(I-\lambda_{0} L\right), x=\beta y$ if and only if $[x, z]=0, z \in\{y\}^{\perp}$.

For convenience we denote $a u_{1}+b u_{2}$ by $(a, b)$. With this convention

$$
P T(\lambda,(a, b)+f(\lambda, a, b))=\left(T_{1}(\lambda, a, b), T_{2}(\lambda, a, b)\right),
$$

where $T_{1}=\left[P T, u_{1}\right]$ and $T_{2}=\left[P T, u_{2}\right]$.

For each $(a, b) \neq(0,0),(b,-a)$ is a basis of $\{(a, b)\}^{\perp}$. Therefore, $\operatorname{PT}(\lambda,(a, b)+f(\lambda, a, b))=\beta(a, b)$ if and only if 


$$
b T_{1}(\lambda, a, b)-a T_{2}(\lambda, a, b)=0 .
$$

Choose $c_{0}>0$ so that $\hat{\lambda}(u)$ is defined for all $u,\|u\|_{H} \leq c_{0}$. Let $0<c<c_{0}$. By hypothesis for all $\lambda$ near $\lambda_{0}, c T_{1}(\lambda, 0, c) \geq 0$ and $-c T_{1}(\lambda, 0,-c) \leq 0$. The function $H(a, b)=b T_{1}(\hat{\lambda}(a, b), a, b)-a T_{2}(\hat{\lambda}(a, b), a, b)$ maps the connected set $\left\{u \in N\left(I-\lambda_{0} L\right) \mid\|u\|_{H}=c\right\}$ into $\mathbf{R}$. We have seen that $H(0, c) \geq 0$, and $H(0,-c)$ $\leq 0$. Therefore, there is a point $(\bar{a}, \bar{b})$ of norm $c$ such that $H(\bar{a}, \bar{b})=0$. Since $\overline{(\hat{\lambda}}(\bar{a}, \bar{b}),(\bar{a}, \bar{b}))$ satisfies equation (3.2) we obtain the equation $P T(\bar{\lambda}, \bar{u}+f(\bar{\lambda}, \bar{u}))$ $=\underline{\beta} \bar{u}$ where $\bar{u}=(\bar{a}, \bar{b}),\|\bar{u}\|_{H}=c$ and $\bar{\lambda}=\hat{\lambda}(\bar{u})$. By Corollary $1.9(\bar{\lambda}, \bar{u}+$ $f(\bar{\lambda}, \bar{u}))$ is a solution of equation (3.1). For each small $c$, there is a solution $\left(\lambda_{c}, u_{c}+f\left(\lambda_{c}, u_{c}\right)\right)$ of equation (3.1). $\left\|u_{c}\right\|_{H}=c$ and $\lambda_{c}=\hat{\lambda}\left(u_{c}\right)$. Therefore, by the continuity of $\hat{\lambda}(u), \lambda_{0}$ is a point of bifurcation of equation (3.1).

\section{PART IV. COUNTEREXAMPLES}

4.1. In this section we give three examples to demonstrate that when some of our hypotheses are removed many of the results of $\$ 2.1$ are not valid. In all of our examples, $T$ depends linearly on $\lambda, \operatorname{dim} N\left(I-\lambda_{0} L\right)=1, T \not 0$ and $T$ satisfies the hypotheses of Theorem 2.1.

Example 1. If $\lambda_{0}$ is not a simple eigenvalue of $L$ then $\lambda_{0}$ may fail to be a bifurcation point of equation (2.2) with respect to 0 .

Consider the system of equations in $\mathbf{R}$ :

$$
\begin{aligned}
x-\lambda\left(x+y+y^{3}\right) & =0, \\
y-\lambda\left(y-x^{3}\right) & =0 .
\end{aligned}
$$

Here $T$ is an odd operator defined by $T(x, y)=\left(y^{3},-x^{3}\right), \lambda_{0}=1$ is an eigenvalue of $L$. A computation will show $\operatorname{dim} N\left(I-\lambda_{0} L\right)=1$ but $\lambda_{0}$ is not simple. In addition, $T$ satisfies the hypotheses of Theorem 2.1 and is real analytic. This system has no solutions for $\lambda$ near $\lambda_{0}=1$ other than $x=y=0$. To see this, suppose $(\lambda, x, y)$ is a solution. Multiplying equation (4.1) by $y$ and equation (4.2) by $x$, and subtracting we get $\lambda\left(y^{2}+y^{4}+x^{4}\right)=0$. Therefore, $x=y=0$.

Example 2. In this example $L+T$ is a gradient operator, $T$ is real analytic, $\lambda_{0}$ is a simple eigenvalue and $I-\lambda_{0} L$ is a Fredholm operator of index 0 . However, though $\lambda_{0}$ is a bifurcation point, $\lambda_{0}$ is the only eigenvalue of the nonlinear problem for $x$ of small norm.

Consider the equations in $\mathbf{R}$,

$$
\begin{aligned}
x-\lambda\left(-2 x-x^{3}\right) & =0, \\
y-\lambda y & =0 .
\end{aligned}
$$

$L$ and $T$ are as indicated above and $\lambda_{0}=1$ is a simple eigenvalue of $L$. Equation (4.3) has no solutions other than $x=0$ for $\lambda$ near 1 . Suppose $x \neq 0$ is a solution of equation (4.3). Then $(1+2 \lambda) x+\lambda x^{3}=0$. Dividing by $x$ and then by 
$\lambda$ we get $\lambda^{-1}(1+2 \lambda)+x^{2}=0$. But this equation has no real solution since $\lambda^{-1}(1+2 \lambda)>0$. On the other hand, equation (4.4) has no solution other than $y=0$ if $\lambda \neq 1 . \lambda_{0}$ is thus the only eigenvalue for small $|x|$.

Example 3. Here $T$ is $n$ times continuously differentiable, $L$ and $T$ satisfy the hypotheses of Theorem 2.1 , and $\lambda_{0}$ is not the only eigenvalue, yet there is one eigenvalue with infinitely many (nonlinear) eigenfunctions $x$. Moreover, for each integer $N$, there exists a sequence of eigenvalues, different from $\lambda_{0}$, such that each eigenvalue has at least $N$ eigenvectors and the eigenvalues and eigenvectors converge to $\lambda_{0}$ and 0 respectively.

Consider the equation

$$
x-\lambda\left(x+x^{m} \sin \left(x^{-1}\right)\right)=0
$$

for $x \in \mathbf{R} . x^{m} \sin \left(x^{-1}\right)$ is $n$ times continuously differentiable for $m=2 n+1$. Clearly the hypotheses of Theorem 2.1 are satisfied and $\lambda_{0}=1$ is a simple eigenvalue of the linearized part. Dividing (4.5) by $x$ we get

$$
(1-\lambda)+x^{m-1} \sin \left(x^{-1}\right)=0 \text {. }
$$

Since $x^{m-1} \sin \left(x^{-1}\right) \notin 0, \lambda_{0}$ is not the only eigenvalue. For $\lambda=\lambda_{0}$ there correspond infinitely many solutions of small norm.

Let $N$ be an integer. Then since $\sin \left(x^{-1}\right)$ oscillates about 0 , we can find a sequence $\left\{\lambda_{n}\right\}, \lambda_{n} \rightarrow \lambda_{0}$, such that for each $\lambda_{n}$ there correspond $N$ distinct values $x_{n_{1}}, \cdots, x_{n_{N}}$ and each $\left(\lambda_{n}, x_{n_{i}}\right)$ satisfies equation (4.6) and $x_{n_{i}} \rightarrow 0$.

Since solutions of equation (4.6) are also solutions of equation (4.5) we have shown the existence of the sequence described above.

\section{PART V. APPENDIX}

We will now give the proofs of those theorems cited in previous sections without proof.

Theorem 5.1. Let $L$ be a bounded linear operator mapping a Banach space $X$ into itself. Suppose $N(L)$ is finite-dimensional. Then $X=N(L) \oplus R(L)$ if and only if $L$ is a Fredbolm operator of index zero and $N(L) \cap R(L)=\{0\}$.

Proof. We denote the space of all bounded linear functionals on $X$ by $X^{\prime}$, the adjoint of $L$ by $L^{\prime}$ and for any subspace $N, N^{\perp}$ is the set of annihilators of $N$.

Suppose $X=N(L) \oplus R(L)$. Then $R(L)$ is closed and $R(L)^{\perp}=N\left(L^{\prime}\right)$. Let $m=\operatorname{dim} N(L)$. Then the dimension of the dual space $N(L)^{\prime}$ of $N(L)$ must also be be $m$. But $R(L)^{\perp}$ is equivalent to $N(L)^{\prime}$. Hence $\operatorname{dim} N\left(L^{\prime}\right)=m$ and $L$ is a Fredholm operator of index 0 .

Suppose now $L$ is Fredholm and ind $(L)=0$. Let $m=\operatorname{dim} N(L)=$ $\operatorname{dim} N\left(L^{\prime}\right) . N(L) \oplus R(L)$ is closed. Suppose $X \neq N(L) \oplus R(L)$. Then there is a 
nonzero vector $x_{0} \in X$ such that $x_{0} \not k N(L) \oplus R(L)$. Let $x_{1}, x_{2}, \cdots, x_{m}$ be a basis of $N(L)$, By the Hahn-Banach theorem there exist $m+1$ bounded linear functionals $y_{0}, y_{1}, \cdots, y_{m}$ such that for $0 \leq i, j \leq m ;\left[x_{i}, y_{j}\right]=0$ for $i \neq j$, $\left[x_{i}, y_{i}\right]=1$ and $\left[x, y_{i}\right]=0$ for $x \in R(L)$. The $y_{i}$ are linearly independent elements of $R(L)^{\perp}$ and $R(L)^{\perp}=N\left(L^{\prime}\right)$. However, $\operatorname{dim} N\left(L^{\prime}\right)=m$ so there cannot be $m+1$ linearly independent vectors in $N\left(L^{\prime}\right)$. Therefore $X=N(L) \oplus R(L)$.

We now prove Lemma 1.5 .

To show statement (i), choose $\epsilon>0$. By hypothesis there is a neighborhood $A$ of $\left(x_{0}, 0\right)$ such that if $(x, y) \in A$ then $(x, t y) \in A$ for $0 \leq t \leq 1$ and

$$
\sup _{(x, y) \in A}\left\|D_{2} T(x, y)\right\| \leq c<\infty .
$$

Let $\delta=\epsilon / c$. Then for any $(x, y) \in A$ with $\|y\| \leq \delta$ we have by inequality (2.17)

$$
\begin{aligned}
\|T(x, y)\| & =\|T(x, y)-T(x, 0)\| \leq\|y\| \sup _{0 \leq t \leq 1}\left\|D_{2} T(x, t y)\right\| \\
& \leq\|y\| c \leq \delta c \leq(\epsilon / c) c=\epsilon .
\end{aligned}
$$

To show statement (ii), let $A=N_{1} \times N_{2}$ where $N_{1}$ is the neighborhood of $x_{0}$ specified in the hypothesis of (ii); $N_{2}$ is a neighborhood of $y=0$ such that if $y \in N_{2}$, then $t y \in N_{2}$ for $0 \leq t \leq 1$; and $N_{1} \times N_{2}$ is in the domain of $T$. Choose $\epsilon>0$. There exists a $\delta>0$ such that $\left\|D_{2} T(x, y)\right\|<\epsilon$ for $(x, y) \in A$ with $\|y\| \leq \delta$. Hence for $(x, y) \in A$ with $\|y\| \leq \delta$,

$$
\|T(x, y)\|=\|T(x, y)-T(x, 0)\| \leq\|y\| \sup _{0 \leq t \leq 1}\left\|D_{2} T(x, t y)\right\| \leq\|y\| \epsilon .
$$

Therefore, $\|T(x, y)\|=o(\|y\|)$.

\section{BIBLIOGRAPHY}

1. Melvyn S. Berger, "A bifurcation theory for nonlinear elliptic partial differential equations and related systems, " in Bifurcation theory and nonlinear eigenvalue problems, Joseph B. Keller and Stuart Antman (Editors), Benjamin, New York, 1969.

2. Melvyn S. Berger and M. S. Berger, Perspectives in nonlinearity. An introduction to nonlinear analysis, Benjamin, New York, 1968. MR $40 \# 4971$.

3. J. Dieudonné, Foundations of modern analys is, Pure and Appl. Math., vol. 10, Academic Press, New York, 1960. MR 22 \#11074.

4. M. A. Krasnosel'skir, Topological methods in the theory of nonlinear integral equations, GITTL, Moscow, 1956; English transl., Macmillan, New York, 1964. MR $20 \# 3464$; MR $28 \# 2414$.

5. - Positive solutions of operator equations, Fizmatgiz, Moscow, 1962; English transl., Noordhoff, Groningen, 1964. MR $26 \# 2862$; MR 31 \#6107.

6. L. A. Ljusternik and S. L. Sobolev, Elements of functional analysis, GITTL, Moscow, 1951; English transl., Ungar, New York, 1961. MR 25 \#5361.

7. M. M. Vaìnberg and V. A. Trenogin, The Lyapunov-Schmidt methods in the theory of non-linear equations and their subsequent development, Uspehi Mat. Nauk 17 (1962), no. 2 (104), 13-75 = Russian Math. Surveys 17 (1962), no. 2, 1-60. MR $27 \# 4071$.

BELFER GRADUATE SCHOOL OF SCIENCE, YESHIVA UNIVERSITY, NEW YORK, NEW YORK 10033

DEPARTMENT OF MATHEMATICS, BROOKLYN COLLEGE, BROOKLYN, NEW YORK 11210

Current address: Department of Mathematics, University of Negev, P.O.B. 2053, Beer-Sheva, Israel 